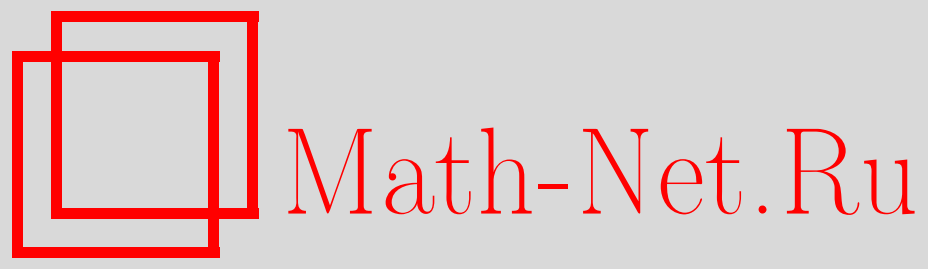

Д. А. Попов, Явная формула для функции распределения собственных значений оператора Лапласа на компактной римановой поверхности рода $g>1$, Функи. анализ и его прил., 2012, том 46, выпуск 2, 66-82

DOI: https://doi.org/10.4213/faa3073

Использование Общероссийского математического портала Math$\mathrm{Net.Ru}$ подразумевает, что вы прочитали и согласны с пользовательским соглашением

http://www . mathnet.ru/rus/agreement

Параметры загрузки:

IP: 54.197 .217 .227

26 апреля 2023 г., 11:47:48

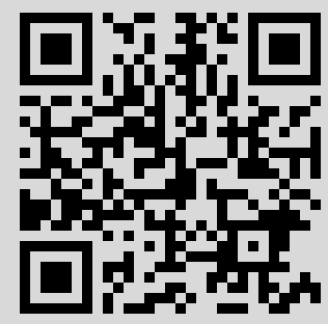




\title{
Явная формула для функции распределения собственных значений оператора Лапласа на компактной римановой поверхности рода $g>1$
}

\author{
(c) 2012. Д. А. Попов
}

Памяти В. И. Арнолъда

Предполагается, что на компактной римановой поверхности рода $g>1$ задана стандартная риманова метрика постоянной кривизны $K=-1$. При этом условии для некоторого класса строго гиперболических фуксовых групп получено явное выражение для функции распределения собственных значений оператора Лапласа в виде ряда по нулям дзета-функции Сельберга.

\section{$\S 1$. Введение и формулировка результата}

В теории чисел явными называются формулы вида

$$
\sum_{n \leqslant x} f(n)=\sum_{\rho} g(x, \rho)+R(x)
$$

где $f$ - некоторая арифметическая функция, а суммирование в правой части этого равенства ведется по нулям $\rho$ некоторой аналитической функции. Относительно функции $R(x)$ предполагается, что она известна или известна ее достаточно точная оценка. При этом $R(x)$ не вносит существенного вклада в асимптотику рассматриваемой суммы при $x \rightarrow \infty$. Первым примером явной формулы была формула Римана для числа $\pi(x)$ простых чисел, меньших $x$ $([1],[2])$. Типичным и самым известным примером является формула Мангольдта для функции Чебышёва [3]

$$
\Psi(x)=\sum_{n \leqslant x} \Lambda(n), \quad \Lambda(n)= \begin{cases}\ln p, & n=p^{k} \\ 0, & n \neq p^{k}\end{cases}
$$

Эта формула имеет вид

$$
\Psi_{0}(x)=x-\sum_{\rho} \frac{x^{\rho}}{\rho}-\frac{\zeta^{\prime}(0)}{\zeta(0)}-\frac{1}{2} \ln \left(1-x^{-2}\right), \quad \Psi_{0}(x)=\frac{1}{2}[\Psi(x+0)+\Psi(x-0)],
$$

и суммирование в ней ведется по нетривиальным нулям дзета-функции Римана. С выводом и различными видами явных формул можно ознакомиться по работам [1]-[7].

Формула Сельберга и связанная с ней дзета-функция Сельберга позволили найти явную формулу для функции $\Psi_{\Gamma}(x)$, связанной с функцией распределения длин замкнутых геодезических на компактных римановых поверхностях рода $g>1$ с метрикой постоянной кривизны $K=-1$. Эта формула будет приведена в 4 . 
В настоящей работе при тех же предположениях будет получена явная формула для функции распределения собственных значений оператора Лапласа.

Приведем некоторые сведения об операторе Лапласа и формуле Сельберга. При этом будем следовать работам [8]-[10] и принятым там обозначениям. Пусть $F=\Gamma \backslash H-$ компактная риманова поверхность рода $g>1$. Здесь $H-$ верхняя полуплоскость и $Г$ - строго гиперболическая (не содержащая эллиптических и параболических элементов) фуксова группа первого рода. Верхняя полуплоскость $H(y>0)$ рассматривается как плоскость Лобачевского с метрикой $d s^{2}=y^{-2}\left(d x^{2}+d y^{2}\right)$. Определенный на гладких функциях оператор Лапласа $\Delta=y^{2}\left(\partial^{2} / \partial x^{2}+\partial^{2} / \partial y^{2}\right)$ опускается на $F$ и отождествляется с оператором на $H$, действующим в пространстве автоморфных функций $f(f(a z)=f(z)$ для любого $a \in \Gamma$ ). Так определенный оператор отрицательно определен и допускает расширение до самосопряженного оператора $L$ в $L_{2}(F)$. Оператор $L$ имеет чисто дискретный спектр $\left\{\lambda_{n}\right\}\left(L \varphi_{n}+\lambda_{n} \varphi_{n}=0, \lambda_{n} \geqslant 0\right)$.

Нас будет интересовать функция распределения собственных значений

$$
\mathscr{N}(x)=\#\left\{n: n \geqslant 0, \lambda_{n} \leqslant x\right\}=\sum_{\lambda_{n} \leqslant x} 1,
$$

которые считаются с учетом их кратности.

В соответствии с формулой Вейля

$$
\mathscr{N}(x)=\frac{|F|}{4 \pi} x+S(x),
$$

где $|F|$ - площадь поверхности $F$. В настоящее время относительно асимптотики функции $S(x)$ известно, что

$$
S(x)=O\left(\frac{x^{1 / 2}}{\ln x}\right) .
$$

Имеется гипотеза, что $S(x)=O\left(x^{1 / 4+\varepsilon}\right)$ и даже (см. [12]) $S(x)=O(\sqrt{\ln x})$ для достаточно общей (не арифметической) группы Г. С другой стороны, для некоторого класса арифметических групп $\Gamma=\Gamma(n, p)$ (см. [8]-[10]) Сельбергом доказана $\Omega$-теорема: $S(x)=\Omega\left(x^{1 / 4}(\ln x)^{-1}\right)$, где $\Omega-$ символ Харди и равенство $S(x)=\Omega(f(x))$ означает, что существует последовательность $x_{i} \rightarrow \infty$, такая, что $\left|S\left(x_{i}\right)\right|>C f\left(x_{i}\right)\left(C>0, x_{i}>X, f\left(x_{i}\right)>0\right)$.

Поведение величины $|S(x)|$ в среднем рассматривалось в работе [11], где показано, что

$$
T^{-1} \int_{0}^{T}|S(x)| d x=O\left(T^{1 / 4+\varepsilon}\right) \quad \forall \varepsilon>0 .
$$

Автор благодарен рецензенту за указание на эту работу.

Рассмотрим формулу Сельберга. Введем нужные обозначения. Через $\{P\}$ обозначается множество классов сопряженных элементов группы Г. Так как $\Gamma$ - строго гиперболическая группа, то каждый элемент $a \in \Gamma$ сопряжен в $\operatorname{PSL}(2, \mathbb{R})$ умножению $z \rightarrow \lambda z(z \in H, \lambda>1)$. Величина $\lambda=N(P)$ называется нормой класса $P$. Эта величина имеет простой геометрический смысл, и множество $\{\ln N(P)\}$ совпадает со спектром длин замкнутых геодезических на поверхности $F$. 
Рассмотрим спектр $\left\{\lambda_{n}\right\}$ оператора $L$. У оператора $L$ имеется $M+1$ исключительных собственных значений $\lambda_{n} \in[0,1 / 4), n=0,1, \ldots, M$. Величины $s_{n}$, $\tilde{s}_{n}, r_{n}$ определяются равенствами

$$
\begin{gathered}
\lambda_{n}=s_{n}\left(1-s_{n}\right)=\frac{1}{4}+r_{n}^{2}, \quad \arg r_{n}=0 \text { или }-\pi / 2, \\
s_{n}=\frac{1}{2}+i r_{n}, \quad \tilde{s}_{n}=\frac{1}{2}-i r_{n} .
\end{gathered}
$$

Таким образом, если $n=0,1, \ldots, M$, то

$$
\begin{gathered}
r_{n}=-i \varkappa_{n}, \quad \varkappa_{n}=\sqrt{\frac{1}{4}-\lambda_{n}}, \quad 0<\varkappa_{n} \leqslant \frac{1}{2}, \\
s_{n}=\frac{1}{2}+\varkappa_{n}, \quad \tilde{s}_{n}=\frac{1}{2}-\varkappa_{n}, \quad \tilde{s}_{0}=0 .
\end{gathered}
$$

Через $h$ обозначается любая функция, удовлетворяющая следующим условиям:

1) $h(r)=h(-r)$,

2) $h$ голоморфна в полосе $|\operatorname{Im} r| \leqslant 1 / 2+\varepsilon, \varepsilon>0$,

3) $h(r)=O\left(1+|r|^{2}\right)^{-1-\varepsilon}$ в этой полосе.

В этих обозначениях формула Сельберга для строго гиперболических групп Г имеет следующий вид:

$$
\sum_{r_{n}} h\left(r_{n}\right)=\frac{|F|}{2 \pi} \int_{-\infty}^{+\infty} r \operatorname{th} \pi r h(r) d r+2 \sum_{\{P\}} \frac{\ln N\left(P_{0}\right)}{N(P)^{1 / 2}-N(P)^{-1 / 2}} g(\ln N(P)) .
$$

В этой формуле

$$
g(u)=\frac{1}{2 \pi} \int_{-\infty}^{+\infty} e^{-i r u} h(r) d r
$$

и $P_{0}$ - отвечающий $P$ примитивный класс сопряженных элементов. Множество таких классов обозначается через $\left\{P_{0}\right\}$. Дзета-функция Сельберга $Z(s)(s=$ $\sigma+i t)$ при $\sigma>1$ определяется равенством

$$
Z(s)=\prod_{\left\{P_{0}\right\}} \prod_{k=0}^{\infty}\left(1-N\left(P_{0}\right)^{-s-k}\right) .
$$

Эта функция допускает аналитическое продолжение на всю комплексную плоскость как целая функция порядка 2. Она имеет «тривиальные» нули в точках $s=-k, k=-1,0,1,2, \ldots$ и «нетривиальные» нули в точках $s=\frac{1}{2}+i r_{n}$ с кратностью, равной кратности соответствующего собственного значения. Нетривиальные нули функции $Z(s)$ лежат на прямой $\sigma=1 / 2$, т. е. для $Z(s)$ имеет место гипотеза Римана.

Аналогом функции Чебышёва $\Psi(x)$ (см. (1.1)) является функция

$$
\Psi^{\Gamma}(x)=\sum_{N(P) \leqslant x} \Lambda^{\Gamma}(P),
$$

где $\Lambda^{\Gamma}$ - аналог функции Мангольдта:

$$
\Lambda^{\Gamma}(P)=\frac{\ln N\left(P_{0}\right)}{1-N(P)^{-1}} .
$$


Аналогом равенства

$$
\frac{\zeta^{\prime}(s)}{\zeta(s)}=-\sum_{n=1}^{\infty} \frac{\Lambda(n)}{n^{s}} \quad(\sigma>1)
$$

является равенство

$$
\frac{Z^{\prime}(s)}{Z(s)}=\sum_{\{P\}} \frac{\Lambda^{\Gamma}(P)}{N(P)^{s}} \quad(\sigma>1)
$$

по существу определяющее функцию $Z(s)$.

Цель настоящей работы состоит в том, чтобы вывести явную формулу для величины

$$
\mathscr{N}\left(t^{2}+1 / 4\right)=\sum_{\lambda_{n} \leqslant t^{2}+1 / 4} 1
$$

в виде некоторого ряда по нулям функции $Z(s)$. Так как нетривиальные нули функции $Z(s)$ прямо связаны с собственными значениями, то такая формула имеет вид тождества, имеющего место на спектре $\left\{\lambda_{n}\right\}$.

Везде ниже через $C$ обозначаются абсолютные константы, а через $C_{\Gamma}-$ константы, зависящие только от группы $\Gamma$, и запись $f(t)=O_{\Gamma}(g(t))$ означает, что константа в $O_{\Gamma}(\cdot)$ зависит только от группы $Г$.

Сформулируем основной результат. Введем ограничение на класс рассматриваемых групп. Будем говорить, что группа Г удовлетворяет условию $(\eta, q)$, если существуют константы $\eta>0, q$, такие, что при $N(P) \neq N\left(P^{\prime}\right)$ выполняется неравенство

$$
\left|N(P)-N\left(P^{\prime}\right)\right| \geqslant \eta N(P)^{-q} .
$$

Из результатов работы [13] (см. также [14]) следует, что условие (1.9) с $q=0$ выполняется для группы $\Gamma$, происходящей из кватернионной алгебры.

Введем обозначения

$$
B_{0}=\min _{\{P\}} N(P), \quad b_{0}=\ln B_{0}, \quad B_{0}>1, b_{0}>0 .
$$

Теорема. Пусть Г-строго гиперболическая группа, для которой въполняется условие $(\eta, q)$. Тогда для любого $t \geqslant 3, t \notin\left\{r_{n}\right\}$,

$\mathscr{N}\left(t^{2}+1 / 4\right)=\frac{|F|}{4 \pi} t^{2}+\frac{1}{\pi} \sum_{r_{n} \geqslant 0}\left[\operatorname{sgn}\left(r_{n}-t\right) \operatorname{si}\left(\left|r_{n}-t\right| b_{0}\right)-\operatorname{si}\left(\left(r_{n}+t\right) b_{0}\right)-\beta_{n}\right]+F^{\Gamma}(t)$.

В этой формуле

$$
\beta_{n}=-\frac{r_{n} \cos \left(r_{n}-t\right) b_{0}}{b_{0}\left(r_{n}^{2}+1 / 4\right)}+\frac{1}{2} \frac{\sin \left(r_{n}-t\right) b_{0}}{b_{0}\left(r_{n}^{2}+1 / 4\right)}+\frac{r_{n} \cos \left(r_{n}+t\right) b_{0}}{b_{0}\left(r_{n}^{2}+1 / 4\right)}-\frac{1}{2} \frac{\sin \left(r_{n}+t\right) b_{0}}{b_{0}\left(r_{n}^{2}+1 / 4\right)}
$$

u $F^{\Gamma}$ - ограниченная непрерывная функиия, допускающая при $t \rightarrow \infty$ асимnтотическое разложение вида

$$
F^{\Gamma}(t)=C_{0}^{\Gamma}+\frac{C_{1}^{\Gamma}}{t}+\frac{C_{2}^{\Gamma}}{t^{2}}+\cdots
$$

Явный вид функции $F^{\Gamma}$ будет указан в $\S 4$. 
В формуле (1.11) и ниже используются стандартные обозначения ([15], [16])

$$
\text { si } x=\int_{\infty}^{x} \frac{\sin t}{t} d t=\operatorname{Si} x-\frac{\pi}{2}, \quad \text { si } x+\operatorname{si}(-x)=-\pi .
$$

В ходе доказательства теоремы в $\S 5$ будет доказано

Предложение. Если группа Г удовлетворяет условиям теоремъ, то сходится ряд

$$
\sum_{r_{n} \geqslant 0} \frac{\cos r_{n} b_{0}}{r_{n}^{2}+1 / 4}=A^{\Gamma}\left(b_{0}\right)<\infty .
$$

Теорема утверждает, что на множестве $\left\{\lambda_{n}\right\}$ для любого $t \geqslant 3$ имеет место равенство

$$
\sum_{\lambda_{n} \leqslant t^{2}+1 / 4} 1=f\left(t \mid \lambda_{1}, \lambda_{2}, \ldots\right),
$$

где $f\left(t \mid \lambda_{1}, \lambda_{2}, \ldots\right)$ - правая часть равенства (1.11), и эта формула является аналогом формулы Харди-Вороного для проблемы круга (см. [8], [10]). Заметим, что, выбирая различные функции $h$ в формуле (1.4), можно получить и другие равенства вида (1.13).

\section{§2. Двусторонняя оценка функции распределения}

Рассмотрим двупараметрическое семейство $\left\{h_{\alpha, T}\right\}, \alpha>0, T>0$, функций

$$
h_{\alpha, T}(r)=\int_{-\infty}^{\infty} \chi_{T}(s) \delta_{\alpha}(r-s) d s .
$$

В этой формуле

$$
\chi_{T}(r)=\left\{\begin{array}{ll}
1, & |r|<T, \\
0, & |r|>T,
\end{array} \quad \delta_{\alpha}(r)=\sqrt{\frac{\alpha}{\pi}} e^{-\alpha r^{2}}, \quad \int_{-\infty}^{+\infty} \delta_{\alpha}(r) d r=1 .\right.
$$

Такое семейство функций в связи с формулой Сельберга рассматривалось в работе [17]. Ниже будут использоваться стандартные обозначения ([15], [16])

$$
\operatorname{erf} z=\frac{2}{\sqrt{\pi}} \int_{0}^{z} e^{-t^{2}} d t, \quad \operatorname{erfc} z=1-\operatorname{erf} z .
$$

Согласно [13], при $x \geqslant 0$ имеет место двусторонняя оценка

$$
\frac{2}{\sqrt{\pi}} \frac{e^{-x^{2}}}{x+\sqrt{x^{2}+2}} \leqslant \operatorname{erfc} x \leqslant \frac{2}{\sqrt{\pi}} \frac{e^{-x^{2}}}{x+\sqrt{x^{2}+4 / \pi}} .
$$

Функция erf $z$ целая и нечетная, и при $|\arg z| \leqslant \pi / 4$ асимптотическое разложение

$$
\operatorname{erfc} z=\frac{e^{-z^{2}}}{\sqrt{\pi} z}\left(1+\sum_{m=1}^{\infty}(-1)^{m} \frac{1 \cdot 3 \cdots(2 m-1)}{\left(2 z^{2}\right)^{m}}\right)
$$

является обертывающим.

Прямо из определения (2.1) следует, что

$$
h_{\alpha, T}(r)=\frac{1}{2}[\operatorname{erf}(\sqrt{\alpha}(r+T))+\operatorname{erf}(\sqrt{\alpha}(T-r))] .
$$


Учитывая указанные свойства функций erf, erfc, получаем, что

$$
h_{\alpha, T}(r)=h_{\alpha, T}(-r), \quad \frac{d h_{\alpha, T}(r)}{d r} \leqslant 0 \quad(r \geqslant 0), \quad 0<h_{\alpha, T}(r)<1
$$

и имеют место оценки

$$
\begin{array}{r}
h_{\alpha, T}(r) \leqslant C \frac{e^{-\alpha(r-T)^{2}}}{\sqrt{\alpha}|r-T|} \quad(C<1, r>T), \\
1-h_{\alpha, T}(r) \leqslant C \frac{e^{-\alpha(r-T)^{2}}}{\sqrt{\alpha}|T-r|} \quad(C<1, T>r) .
\end{array}
$$

Из приведенных результатов следует, что любая функция $h \in\left\{h_{\alpha, T}\right\}$ удовлетворяет условиям (1.3). При $h=h_{\alpha, T}$ формула Сельберга (1.4) имеет вид

$$
\sum_{n \geqslant 0} h_{\alpha, T}\left(r_{n}\right)=\frac{|F|}{4 \pi} \int_{-\infty}^{+\infty} r \operatorname{th} \pi r h_{\alpha, T}(r) d r+S_{\alpha, T}^{\Gamma} .
$$

Здесь и ниже

$$
S_{\alpha, T}^{\Gamma}=\sum_{\{P\}} \frac{\ln N\left(P_{0}\right)}{N(P)^{1 / 2}-N(P)^{-1 / 2}} g_{\alpha, T}(\ln N(P)),
$$

и в этой формуле

$$
g_{\alpha, T}(u)=\frac{1}{2 \pi} \int_{-\infty}^{+\infty} e^{-i r u} h_{\alpha, T}(r) d r=\frac{1}{\pi} \frac{\sin T u}{u} e^{-u^{2} /(4 \alpha)} .
$$

В этом параграфе рассматривается левая часть равенства (2.5) и основным результатом является

\section{Лемма 1. Пусть}

$$
\gamma>0, \quad \sigma \geqslant \gamma+3, \quad t \geqslant 3 .
$$

Тогда для любых таких $\gamma, \sigma, t$ при

$$
\alpha=t^{2 \gamma}\left(\ln t^{\sigma}\right), \quad T_{ \pm}=t \pm \delta, \quad \delta=t^{-\gamma}
$$

имеют место неравенства

$$
\begin{aligned}
& \mathscr{N}\left(t^{2}+1 / 4\right) \geqslant \frac{|F|}{4 \pi} t^{2}+F_{0}^{\Gamma}(t)+S_{\alpha, T_{-}}^{\Gamma}+\Delta \mathscr{N}^{-}(t), \\
& \mathscr{N}\left(t^{2}+1 / 4\right) \leqslant \frac{|F|}{4 \pi} t^{2}+F_{0}^{\Gamma}(t)+S_{\alpha, T_{+}}^{\Gamma}+\Delta \mathscr{N}^{+}(t) .
\end{aligned}
$$

В этих формулах

$$
F_{0}^{\Gamma}(t)=-\frac{|F|}{2 \pi} \int_{0}^{t} r(1-\operatorname{th} \pi r) d r
$$

и для функиий $\Delta \mathscr{N}^{ \pm}(t)$ выполняется оценка

$$
\left|\Delta \mathscr{N}^{ \pm}(t)\right| \leqslant C_{\Gamma} t^{1-\gamma} .
$$


Доказательство. Введем обозначения

$$
\begin{gathered}
H_{\alpha, T}=\sum_{r_{n} \geqslant 0} h_{\alpha, T}\left(r_{n}\right), \quad \Sigma_{M}(\alpha, T)=\sum_{0<\varkappa_{n} \leqslant 1 / 4} h_{\alpha, T}\left(-i \varkappa_{n}\right), \\
F_{\alpha, T}(t)=\int_{-\infty}^{+\infty} r \operatorname{th} \pi r h_{\alpha, T}(r) d r \quad\left(T=t \pm t^{-\gamma}\right)
\end{gathered}
$$

и запишем формулу Сельберга в виде

$$
H_{\alpha, T}+\Sigma_{M}(\alpha, T)=\frac{|F|}{4 \pi} F_{\alpha, T}(t)+S_{\alpha, T}^{\Gamma} .
$$

Рассмотрим левую часть этого равенства.

Интересующую нас величину $\mathscr{N}\left(t^{2}+1 / 4\right)$ запишем в виде

$$
\mathscr{N}\left(t^{2}+1 / 4\right)=\mathscr{N}_{+}(t)+M+1 .
$$

В этой формуле и ниже

$$
\mathscr{N}_{+}(t)=\#\left\{n: 0 \leqslant r_{n} \leqslant t\right\} .
$$

Используя свойства функции $h_{\alpha, T}$ (см. (2.3)), получим, что

$$
\mathscr{N}_{+}(t) \geqslant H_{\alpha, T_{-}}+\Delta^{-}, \quad \Delta^{-}=\sum_{r_{n}>t} h_{\alpha, T_{-}} .
$$

Оценим величину $\Delta^{-}$. Используя оценки $(2.4)$, имеем

$$
\Delta^{-} \leqslant C \sum_{k=0}^{\infty} f(t+k \Delta) \sum_{t+k \Delta \leqslant r_{n} \leqslant t+(k+1) \Delta} 1, \quad f(x)=\frac{e^{-\alpha\left(x-T_{-}\right)^{2}}}{\sqrt{\alpha}\left(x-T_{-}\right)} .
$$

Будем предполагать, что

$$
1<\Delta<t, \quad 2 \alpha \Delta \delta>1 \quad\left(\delta=t^{-\gamma}\right) .
$$

Можно считать, например, что $\Delta=2$. В силу формулы Вейля

$$
\sum_{t+k \Delta \leqslant r_{n} \leqslant t+(k+1) \Delta} 1=O_{\Gamma}\left(t \Delta+(k+1) \Delta^{2}\right) .
$$

Учитывая это, с помощью неравенства (2.15) получим оценку

$$
\Delta^{-} \leqslant C_{\Gamma} \frac{e^{-\alpha \delta^{2}}}{\sqrt{\alpha} \delta} t \Delta
$$

Теперь запишем величину (2.14) в виде

$$
\mathscr{N}_{+}(t)=\sum_{0 \leqslant r_{n} \leqslant t} h_{\alpha, T}\left(r_{n}\right)+\Delta^{+}, \quad \Delta^{+}=\sum_{0 \leqslant r_{n} \leqslant t}\left(1-h_{\alpha, T}\left(r_{n}\right)\right) .
$$

Следовательно,

$$
\mathscr{N}_{+}(t) \leqslant H_{\alpha, T_{+}}+\Delta^{+} \text {. }
$$

Используя оценки (2.4) и то, что в силу формулы Вейля $\sum_{r_{n} \leqslant t} 1 \leqslant C_{\Gamma} t^{2}$, получаем оценку

$$
\Delta^{+} \leqslant C_{\Gamma} \frac{e^{-\alpha \delta^{2}}}{\sqrt{\alpha} \delta} t^{2}
$$


Таким образом, при выполнении условий (2.16) справедлива двусторонняя оценка

$$
M+1+H_{\alpha, T_{-}}-\Delta^{-} \leqslant \mathscr{N}\left(t^{2}+1 / 4\right) \leqslant M+1+H_{\alpha, T_{+}}+\Delta^{+}
$$

вместе с оценками (2.17), (2.18).

Рассмотрим сумму

$$
\Sigma_{M}(\alpha, T)=\sum_{0<\varkappa_{n} \leqslant 1 / 4} h_{\alpha, T}\left(-i \varkappa_{n}\right)
$$

TaK как

$$
\begin{aligned}
h_{\alpha, T}\left(-i \varkappa_{n}\right) & =1-\Delta h_{\alpha, T}\left(\varkappa_{n}\right), \\
\Delta h_{\alpha, T}\left(\varkappa_{n}\right) & =\frac{1}{2} \operatorname{erfc}\left(T+i \varkappa_{n}\right)+\frac{1}{2} \operatorname{erfc}\left(T-i \varkappa_{n}\right),
\end{aligned}
$$

то из равенства (2.20) следует, что

$$
\Sigma_{M}(\alpha, T)=M+1+\Delta_{M}(\alpha, T), \quad \Delta_{M}(\alpha, T)=\sum_{0<\varkappa_{n} \leqslant 1 / 4} \Delta h_{\alpha, T}\left(\varkappa_{n}\right) .
$$

При $\sqrt{\alpha} t>1$, используя $(2.2)$, получим оценку

$$
\Delta_{M}\left(\alpha, T_{ \pm}\right) \leqslant C(M+1) \frac{e^{-\alpha\left(T_{ \pm}-1 / 16\right)}}{\sqrt{\alpha} T_{ \pm}} .
$$

Подставляя выражение для $M+1$, полученное из $(2.21)$, в $(2.19)$, запишем эту двустороннюю оценку в виде

$$
\begin{aligned}
& \Sigma_{M}\left(\alpha, T_{-}\right)+H_{\alpha, T_{-}}+\Delta_{M}\left(\alpha, T_{-}\right)-\Delta_{-} \\
& \quad \leqslant \mathscr{N}\left(t^{2}+1 / 4\right) \leqslant \Sigma_{M}\left(\alpha, T_{+}\right)+H_{\alpha, T_{+}}+\Delta_{M}\left(\alpha, T_{+}\right)+\Delta_{+} .
\end{aligned}
$$

Теперь рассмотрим величину $F_{\alpha, T}(t)$ из $(2.12)$ :

$$
\begin{gathered}
F_{\alpha, T}(t)=2 J_{\alpha, T}+2 I_{\alpha, T} \\
J_{\alpha, T}=\int_{0}^{t} r \operatorname{th} \pi r h_{\alpha, T}(r) d r, \quad I_{\alpha, T}=\int_{t}^{\infty} r \operatorname{th} \pi r h_{\alpha, T}(r) d r .
\end{gathered}
$$

Пусть $T=T_{-}=t-\delta$. Так как тогда при $r>T_{-}$

$$
h_{\alpha, T}(r) \leqslant \frac{1}{2} \operatorname{erfc}(\sqrt{\alpha}(r-T)) \leqslant \frac{1}{2 \sqrt{\pi}} \frac{e^{-\alpha(r-T)^{2}}}{\sqrt{\alpha}(r-T)},
$$

TO

Интеграл $J_{\alpha, T}$ запишем в виде

$$
I_{\alpha, T} \leqslant C \frac{t e^{-\alpha \delta^{2}}}{\alpha^{3 / 2} \delta}
$$

$$
J_{\alpha, T}=J_{\alpha, T}^{1}+J_{\alpha, T}^{2},
$$

и в этой формуле при $t=T+\delta$

$$
J_{\alpha, T}^{1}=\int_{0}^{T-\delta} r \operatorname{th} \pi r h_{\alpha, T}(r) d r, \quad J_{\alpha, T}^{2}=\int_{T-\delta}^{T+\delta} r \operatorname{th} \pi r h_{\alpha, T}(r) d r .
$$

Tак как $h_{\alpha, T}(r)<1$, th $\pi r<1$, то

$$
J_{\alpha, T}^{2} \leqslant C T \delta
$$


Рассмотрим интеграл

$$
J_{\alpha, T}^{1}=\int_{0}^{T-\delta} r \operatorname{th} \pi r d r-\int_{0}^{T-\delta}\left(1-h_{\alpha, T}(r)\right) r \operatorname{th} \pi r d r .
$$

Так как при $r<T$

$$
1-h_{\alpha, T}(r) \leqslant \operatorname{erfc}(\sqrt{\alpha}(T-r)) \leqslant C \frac{e^{-\alpha(T-r)^{2}}}{\sqrt{\alpha}(T-r)},
$$

то получаем, что

$$
\int_{0}^{T-\delta}\left(1-h_{\alpha, T}(r)\right) r \operatorname{th} \pi r d r \leqslant C \frac{e^{-\alpha \delta^{2}} t}{\alpha^{3 / 2} \delta^{2}} .
$$

Остается рассмотреть первый интеграл в правой части равенства (2.23). С учетом оценки (2.24) получаем, что

$$
J_{\alpha, T}^{1}=\frac{1}{2} t^{2}-\int_{0}^{t} r(1-\operatorname{th} \pi r) d r+\Delta J_{\alpha, T}^{1}, \quad\left|\Delta J_{\alpha, T}^{1}\right| \leqslant C\left(t \delta+\frac{e^{-\alpha \delta^{2}} t}{\alpha^{3 / 2} \delta^{2}}\right) .
$$

Собирая результаты, имеем

$$
F_{\alpha, T_{-}}=t^{2}-2 \int_{0}^{t}(1-\operatorname{th} \pi r) r d r+\Delta F_{\alpha, T_{-}}, \quad\left|\Delta F_{\alpha, T_{-}}\right| \leqslant C\left(t \delta+\frac{e^{-\alpha \delta^{2}} t}{\alpha^{3 / 2} \delta^{2}}\right) .
$$

Так как $F_{\alpha, T_{+}}(t)=F_{\alpha, T_{-}}\left(t^{\prime}\right), t^{\prime}=t+2 \delta$, то и

$$
F_{\alpha, T_{+}}=t^{2}-2 \int_{0}^{t}(1-\operatorname{th} \pi r) r d r+\Delta F_{\alpha, T_{+}}, \quad\left|\Delta F_{\alpha, T_{+}}\right| \leqslant C\left(t \delta+\frac{e^{-\alpha \delta^{2}} t}{\alpha^{3 / 2} t^{2}}\right) .
$$

Для получения нужных оценок (2.9), (2.10) теперь достаточно в (2.22) воспользоваться формулой Сельберга (2.13), равенствами $(2.25),(2.26)$ и условиями (2.8). Лемма 1 доказана.

\section{§3. Явная формула для функции $\Psi^{\Gamma}$ и ее применение}

Функция $\Psi^{\Gamma}$ была определена в (1.6). Покажем, что сумму (2.6) можно выразить через эту функцию. Для этого выпишем в удобном для нас виде формулу частичного суммирования Абеля [4].

Пусть $\{P\}-$ любое бесконечное счетное множество и заданы три функции

$$
N:\{P\} \rightarrow \mathbb{R}, \quad L:\{P\} \rightarrow \mathbb{C}, \quad f: \mathbb{R} \rightarrow \mathbb{C} .
$$

При этом функция $f$ непрерывно дифференцируема, а функция $N$ ограничена снизу и не ограничена сверху. Рассмотрим сумму

$$
S(x)=\sum_{N_{0} \leqslant N(P) \leqslant x} L(P) f(N(P)) .
$$

Тогда формула Абеля имеет вид

$$
S(x)=B(x) f(x)-\int_{N_{0}}^{x} B(\xi) f^{\prime}(\xi) d \xi, \quad B(\xi)=\sum_{N_{0} \leqslant N(P) \leqslant \xi} L(P) .
$$


При этом если $B(x) f(x) \rightarrow 0$ при $x \rightarrow \infty$, то

$$
\sum_{N(P) \geqslant N_{0}} L(P) f(N(P))=-\int_{N_{0}}^{\infty} B(\xi) f^{\prime}(\xi) d \xi
$$

при условии, что конечна правая или левая часть этого равенства. Формула (3.1) только по виду отличается от формулы, приведенной в книге [4], и прямо следует из нее.

Учитывая определение (1.7) функции $\Lambda^{\Gamma}(P)$, запишем сумму $S_{\alpha, T}^{\Gamma}$ в виде

$$
S_{\alpha, T}^{\Gamma}=\sum_{\{P\}} \Lambda^{\Gamma}(P) N(P)^{-1 / 2} g_{\alpha, T}(\ln N(P)) .
$$

Так как $\Psi^{\Gamma}(x) \leqslant C_{\Gamma} x$, то, учитывая явный вид $(2.7)$ функции $g_{\alpha, T}$, получаем, что можно воспользоваться формулой (3.2), и тогда

$$
S_{\alpha, T}^{\Gamma}=-\int_{B_{0}}^{\infty} \Psi^{\Gamma}(x) G_{\alpha, T}(x) d x
$$

В этой формуле

$$
G_{\alpha, T}(x)=\frac{d}{d x}\left(x^{-1 / 2} g_{\alpha, T}(\ln x)\right)=\frac{e^{-\ln ^{2} x /(4 \alpha)}}{\pi x^{3 / 2} \ln x}[T \cos (T \ln x)-a(x) \sin (T \ln x)] .
$$

Величина $B_{0}$ определена равенством (1.10), и

$$
a(x)=\frac{1}{2}+\frac{1}{\ln x}+\frac{\ln x}{2 \alpha} .
$$

Явная формула для функции $\Psi^{\Gamma}$, доказанная в книге [9], имеет вид

$$
\Psi^{\Gamma}(x)=\Psi_{M}^{\Gamma}(x)+\Psi_{R}^{\Gamma}(x)+\Delta_{R} \Psi^{\Gamma}(x) .
$$

При этом

$$
\Psi_{M}^{\Gamma}(x)=a_{0}^{\Gamma}+a_{1}^{\Gamma} \ln x+(2 g-2) \ln \left(1-x^{-1}\right)+\frac{4 g-2}{x-1}+\sum_{m=0}^{M}\left(\frac{x^{s_{m}}}{s_{m}}+\frac{x^{\tilde{s}_{m}}}{\tilde{s}_{m}}\right) .
$$

Штрих в сумме по $m$ в правой части этого равенства означает, что член с $\tilde{s}_{m}=0$ опущен, $g$ - род поверхности $F$ и константы $a_{0}^{\Gamma}, a_{1}^{\Gamma}$ зависят только от группы $\Gamma$, а величины $s_{m}, \tilde{s}_{m}$ определены в $(1.2)$. Функция $\Psi_{R}^{\Gamma}$ задается равенством

$$
\Psi_{R}^{\Gamma}(x)=\sum_{0 \leqslant r_{n} \leqslant R}\left(\frac{x^{s_{n}}}{s_{n}}+\frac{x^{\tilde{s}_{n}}}{\tilde{s}_{n}}\right)+\frac{|F| x^{1 / 2} \cos (R \ln x)}{\pi \ln x}
$$

и если $R \geqslant \max \left(1000, x^{2}\right), R \notin\left\{r_{n}\right\}, x \notin\{N(P)\}$, то при $R \rightarrow \infty$ справедлива оценка

$$
\Delta_{R} \Psi^{\Gamma}(x)=O_{\Gamma}\left(\frac{1}{d(x)} \frac{x^{2}}{R}\right)+O_{\Gamma}\left(\frac{x^{1 / 2}}{\ln R}\right), \quad d(x)=\min _{\{P\}}|x-N(P)| .
$$

Покажем, что если выполняется условие $(\eta, q)$ (см. $(1.9))$, то для любого $b>$ $q+2$

$$
\Delta_{R} \Psi^{\Gamma}(x)=O_{\Gamma}\left(\frac{x^{b}}{R \eta}\right)+O_{\Gamma}\left(\frac{x^{1 / 2}}{\ln R}\right)
$$


Формула (3.6) с оценкой (3.10) при $x \notin\{N(P)\}$ доказывается так же, как и соответствующая формула с оценкой (3.9). Для этого рассматривается интеграл

$$
J=\frac{1}{2 \pi i} \int_{\mathscr{L}} \frac{Z^{\prime}(s)}{Z(s)} x^{s} \frac{d s}{s},
$$

где $Z(s)$ - дзета-функция Сельберга (см. (1.5), (1.8)) и $\mathscr{L}$ - прямоугольник на плоскости $s=\sigma+i t$ с вершинами в точках $b \pm i R, \pm A+i R$. Используя информацию о нулях функции $Z$, этот интеграл можно вычислить как сумму вычетов. Отличие от доказательства в книге [9] имеется только при вычислении интеграла

$$
I(x)=\frac{1}{2 \pi i} \int_{b-i R}^{b+i R} \frac{Z^{\prime}(s)}{Z(s)} x^{s} \frac{d s}{s}=\sum_{\{P\}} \Lambda^{\Gamma}(P) \frac{1}{2 \pi i} \int_{b-i R}^{b+i R}\left(\frac{x}{N(P)}\right)^{s} \frac{d s}{s} .
$$

Так как функция $\Psi^{\Gamma}(x)$ постоянна при $N(P) \leqslant x<N\left(P^{\prime}\right)$, где число $N\left(P^{\prime}\right)$ следует за $N(P)$ в упорядоченном по величине множестве $\{N(P)\}$, то достаточно рассмотреть случай $x \in\left\{N(Q)+\frac{1}{2} \eta N(Q)^{-q}\right\}(\{Q\}=\{P\})$. Используя стандартную схему рассмотрения таких интегралов (см. [3], [4]), получаем, что

$$
I(x)=\Psi^{\Gamma}(x)+\Delta^{\Gamma} I(x) .
$$

Так как $|\ln (x / N(P))|>C \eta N(P)^{-q-1}$ при нашем выборе $x$, то

$$
\left|\Delta^{\Gamma} I(x)\right| \leqslant C \sum_{\{P\}} \frac{1}{R \eta} \frac{1}{N(P)^{b-q-1}} .
$$

Отсюда с помощью формулы (3.2) и учитывая, что $\sum_{\{P\}} \Lambda^{\Gamma}(P) \leqslant C_{\Gamma} x$, получаем оценку

$$
\left|\Delta^{\Gamma} I(x)\right| \leqslant C_{\Gamma} \frac{x^{b}}{R \eta} .
$$

Дальнейшее доказательство повторяет соответствующую часть доказательства, приведенного в книге [9].

Подставляя полученное выражение для $\Psi^{\Gamma}(x)$ в формулу $(3.3)$, получаем равенство

$$
S_{\alpha, T}^{\Gamma}=S_{M}^{\Gamma}(\alpha, T)+S_{R}^{\Gamma}(\alpha, T)+\Delta_{R} S_{\alpha, T}^{\Gamma},
$$

в котором

$$
\begin{aligned}
S_{M}^{\Gamma}(\alpha, T) & =-\int_{B_{0}}^{\infty} \Psi_{M}^{\Gamma}(x) G_{\alpha, T}(x) d x, \\
S_{R}^{\Gamma}(\alpha, T) & =-\int_{B_{0}}^{\infty} \Psi_{R}^{\Gamma}(x) G_{\alpha, T}(x) d x, \\
\Delta_{R} S_{\alpha, T}^{\Gamma} & =-\int_{B_{0}}^{\infty} \Delta_{R} \Psi^{\Gamma}(x) G_{\alpha, T}(x) d x .
\end{aligned}
$$

Следующая лемма получается при переходе к пределу при $R \rightarrow \infty$ в этих формулах.

Лемма 2. Если выполняются условия теоремы, то имеет место равенство

$$
S_{\alpha, T}^{\Gamma}=S_{M}^{\Gamma}(\alpha, T)+S_{1}^{\Gamma}(\alpha, T),
$$


где величина $S_{M}^{\Gamma}(\alpha, T)$ определена равенствами (3.11), (3.7), (3.4),

$$
S_{1}^{\Gamma}(\alpha, T)=-\sum_{r_{n} \geqslant 0} \int_{B_{0}}^{\infty}\left(\frac{x^{s_{n}}}{s_{n}}+\frac{x^{\tilde{s}_{n}}}{\tilde{s}_{n}}\right) G_{\alpha, T}(x) d x
$$

и этот ряд сходится при $T>2, \alpha>1$.

Доказательство. Используя оценку (3.10) и делая в интеграле (3.13) замену переменных $y=\ln x$, получаем, что

$$
\left|\Delta_{R} S_{\alpha, T}^{\Gamma}\right| \leqslant C_{\Gamma} T\left[\frac{1}{R \eta} \int_{0}^{\infty} e^{-y^{2} /(4 \alpha)+y(b-1 / 2)} d y+\frac{1}{\ln R} \int_{0}^{\infty} e^{-y^{2} /(4 \alpha)} d y\right]
$$

Откуда следует, что

$$
\lim _{R \rightarrow \infty} \Delta_{R} S_{\alpha, T}^{\Gamma}=0
$$

Необходимо еще оценить вклад второго члена в правой части равенства (3.8), т. е. величину

$$
\Delta S_{R}^{\Gamma}(\alpha, T)=-\int_{B_{0}}^{\infty} \frac{|F| x^{1 / 2} \cos (R \ln x)}{\pi \ln x} G_{\alpha, T}(x) d x
$$

Снова переходя к переменной $y=\ln x$ и интегрируя по частям, получаем оценку

$$
\left|\Delta S_{R}^{\Gamma}(\alpha, T)\right| \leqslant C_{\Gamma} \frac{T^{2}}{R}
$$

и, следовательно, $\lim _{R \rightarrow \infty} \Delta S_{R}^{\Gamma}(\alpha, T)=0$. Сходимость ряда (3.15) вытекает из формулы Сельберга. Лемма 2 доказана.

\section{§4. Вклад исключительных собственных значений}

Вкладом исключительных собственных значений мы называем величину $\lim _{\alpha \rightarrow \infty} S_{M}^{\Gamma}(\alpha, T)$. В этом параграфе доказывается

Лемма 3. Имеет место равенство

$$
\lim _{\alpha \rightarrow \infty} S_{M}^{\Gamma}(\alpha, T)=F_{1}^{\Gamma}(T) \quad(T>1)
$$

в котором $F_{1}^{\Gamma}$ - ограниченная функиия, имеющая при $T \rightarrow \infty$ асимптотику вида

$$
F_{1}^{\Gamma}(T)=C_{0}^{\Gamma}+\frac{C_{1}^{\Gamma}}{T}+\frac{C_{2}^{\Gamma}}{T^{2}}+\cdots
$$

Доказательство. Переходя к переменному $y=\ln x$, запишем интеграл (3.11) в виде

$$
S_{M}^{\Gamma}(\alpha, T)=-A_{0}^{\Gamma}(\alpha, T)-\sum_{m=0}^{M}\left[B_{m}^{\Gamma}(\alpha, T)+\widetilde{B}_{m}^{\Gamma}(\alpha, T)\right]
$$


где величины $A_{0}^{\Gamma}(\alpha, T), B_{m}^{\Gamma}(\alpha, T)$ и $\widetilde{B}_{m}^{\Gamma}(\alpha, T)$ определены равенствами

$$
\begin{aligned}
A_{0}^{\Gamma}(\alpha, T)=\int_{b_{0}}^{\infty} \frac{e^{-y^{2} /(4 \alpha)-y / 2}}{\pi y}[T \cos T y-\tilde{a}(y) \sin T y] \\
\quad \times\left[a_{0}^{\Gamma}+a_{1}^{\Gamma} y+(2 g-2) \ln \left(1-e^{-y}\right)+\frac{4 g-2}{e^{y}-1}\right] d y, \\
B_{m}^{\Gamma}(\alpha, T)=\frac{1}{s_{m} \pi} \int_{b_{0}}^{\infty} \frac{e^{-y^{2} /(4 \alpha)-y / 2+s_{m} y}}{y}[T \cos T y-\tilde{a}(y) \sin T y] d y, \\
\widetilde{B}_{m}^{\Gamma}(\alpha, T)=\frac{1}{s_{m} \pi} \int_{b_{0}}^{\infty} \frac{e^{-y^{2} /(4 \alpha)-y / 2+\tilde{s}_{m} y}}{y}[T \cos T y-\tilde{a}(y) \sin T y] d y
\end{aligned}
$$

и в этих формулах (см. (3.5))

$$
\tilde{a}(y)=\frac{1}{2}+\frac{1}{y}+\frac{y}{2 \alpha} .
$$

Напомним, что член с $\tilde{s}_{m}=0$ опущен. Так как $0<\tilde{s}_{m}<1 / 2$, то в интегралах $A_{0}^{\Gamma}(\alpha, T)$ и $\widetilde{B}_{m}^{\Gamma}(\alpha, T)$ можно переходить к пределу при $\alpha \rightarrow \infty$ под знаком интеграла. Обозначим эти пределы соответственно через $A_{0}^{\Gamma}(T)$ и $\widetilde{B}_{m}^{\Gamma}(T)$. Таким образом,

$$
\begin{aligned}
A_{0}^{\Gamma}(T)= & \int_{b_{0}}^{\infty} \frac{e^{-y / 2}}{\pi y}[T \cos T y-\tilde{a}(y) \sin T y] \\
& \times\left[a_{0}^{\Gamma}+a_{1}^{\Gamma} y+(2 g-2) \ln \left(1-e^{-y}\right)+\frac{4 g-2}{e^{y}-1}\right] d y, \\
\widetilde{B}_{m}^{\Gamma}(T)= & \int_{b_{0}}^{\infty} \frac{e^{-y / 2+\tilde{s}_{m} y}}{y}\left[T \cos T y-\left(\frac{1}{2}+\frac{1}{y}\right) \sin T y\right] d y .
\end{aligned}
$$

Функции $A_{0}^{\Gamma}, \widetilde{B}_{m}^{\Gamma}$ - гладкие ограниченные функции от $T$. Интегрируя по частям, получаем для них асимптотические разложения вида (4.2). Осталось рассмотреть интеграл (4.4). Докажем, что существует предел

$$
\lim _{\alpha \rightarrow \infty} B_{m}^{\Gamma}(\alpha, T)=B_{m}^{\Gamma}(T)
$$

и при этом функция $B_{m}^{\Gamma}(T)$ ограничена и имеет асимптотическое разложение вида (4.2). Тогда равенство (4.1) будет доказано и в нем

$$
F_{1}^{\Gamma}(T)=-A_{0}^{\Gamma}(T)-\sum_{m=0}^{M}{ }^{\prime}\left[B_{m}^{\Gamma}(T)+\widetilde{B}_{m}^{\Gamma}(T)\right] .
$$

Трудность состоит в том, что, в отличие от интеграла (4.5), в интеграле (4.4) величина $s_{m}$ удовлетворяет условию $1 / 2<s_{m} \leqslant 1$. Интегралы $B_{m}^{\Gamma}(\alpha, T)$ имеют вид

$$
I^{ \pm}(\beta, T)=\int_{b_{0}}^{\infty} \varphi(u) e^{-\beta u+p u \pm i T u} d u
$$

где $p=s_{m}-1 / 2, T>p, \beta=(4 \alpha)^{-1}$. Вычислим их предел при $\beta \rightarrow 0$. Рассмотрим интеграл $I^{+}(\beta, T)$. Выберем на плоскости $w=u+i v$ контур $\mathscr{L}$ в виде криволинейной трапеции со сторонами $\mathscr{L}_{i}(i=1,2,3,4)\left(\mathscr{L}=\mathscr{L}_{1} \cup \mathscr{L}_{2} \cup \mathscr{L}_{3} \cup \mathscr{L}_{4}\right)$. 
Здесь $\mathscr{L}_{1}=\left[b_{0}, R\right], \mathscr{L}_{2}$ - дуга окружности $|w|=R$, проведенная от точки $w=R$ до пересечения с прямой $v=u, \mathscr{L}_{3}$ - отрезок этой прямой от точки пересечения с дугой $|w|=R$ до точки $w=b_{0}+i b_{0}$ и $\mathscr{L}_{4}$ - отрезок вертикальной прямой, соединяющий точки $w=b_{0}+i b_{0}$ и $w=b_{0}$. Предполагается, что функция $\varphi$ голоморфна внутри контура $\mathscr{L}$ и в этой области $|\varphi(w)|<C|w|^{k}$. Тогда если $\Psi_{+}(w)=\varphi(w) e^{-\beta w^{2}+p w+i T w}$, то

$$
I^{+}(\beta, w)=-\lim _{R \rightarrow \infty} \int_{\mathscr{L}_{3}} \Psi_{+}(w) d w-\int_{\mathscr{L}_{4}} \Psi_{+}(w) d w,
$$

так как $\lim _{R \rightarrow \infty} \int_{\mathscr{L}_{2}} \Psi_{+}(w) d w=0$. Теперь в интегралах, стоящих в правой части этого равенства, можно перейти к пределу при $\beta \rightarrow 0$ под знаком интеграла. Получим, что

$$
\begin{gathered}
\lim _{\beta \rightarrow 0} \lim _{R \rightarrow \infty} \int_{\mathscr{L}_{3}} \Psi_{+}(w) d w=O\left(e^{-T b_{0}}\right), \\
\lim _{\beta \rightarrow 0} \int_{\mathscr{L}_{4}} \Psi_{+}(w) d w=\left.e^{i T b_{0}+p b_{0}} \int_{0}^{b_{0}} e^{-T v+i p v} \varphi\right|_{u=b_{0}} i d v=O(1 / T) .
\end{gathered}
$$

Нужный результат (4.2) получается интегрированием по частям.

Чтобы рассмотреть интеграл $I^{-}(\beta, T)$, надо действовать так же, только вместо контура $\mathscr{L}$ взять его отражение $\mathscr{L}^{*}$ относительно действительной оси. Лемма 3 доказана.

\section{§5. Доказательство теоремы и предложения}

Рассмотрим формулы (2.9), (2.10) и устремим в них $\gamma$ к $\infty$ при фиксированном $t \geqslant 3$. Из леммы 1 следует, что

$$
\begin{aligned}
& \mathscr{N}\left(t^{2}+1 / 4\right) \geqslant \frac{|F|}{4 \pi} t^{2}+F_{0}^{\Gamma}(t)+\lim _{\gamma \rightarrow \infty} S_{\alpha, T_{-}}^{\Gamma}, \\
& \mathscr{N}\left(t^{2}+1 / 4\right) \leqslant \frac{|F|}{4 \pi} t^{2}+F_{0}^{\Gamma}(t)+\lim _{\gamma \rightarrow \infty} S_{\alpha, T_{+}}^{\Gamma} \quad\left(T^{ \pm}=t \pm t^{-\gamma}\right) .
\end{aligned}
$$

Так как $\alpha=t^{2 \gamma}\left(\ln t^{\sigma}\right)$, то $\alpha \rightarrow \infty$ при $\gamma \rightarrow \infty$. Предел $\lim _{\alpha \rightarrow \infty} S_{M}^{\Gamma}(\alpha, T)$ вычислен в лемме 3 , и имеет место формула (3.14). Таким образом, остается вычислить предел $\lim _{\alpha \rightarrow \infty} S_{1}^{\Gamma}(\alpha, T)$, где функция $S_{1}^{\Gamma}(\alpha, T)$ определена равенством (3.15).

Учитывая вид (3.4) функции $G_{\alpha, T}(x)$ и переходя к переменной $y=\ln x$ в интегралах формулы (3.15), получаем

$$
S_{1}^{\Gamma}(\alpha, T)=-\frac{1}{\pi} \sum_{r_{n} \geqslant 0} \frac{J_{n}(\alpha, T)}{r_{n}^{2}+1 / 4},
$$

и в этой формуле

$$
\begin{gathered}
J_{n}(\alpha, T)=\int_{b_{0}}^{\infty} \frac{e^{-y^{2} /(4 \alpha)}}{y}[\cos T y-\tilde{a}(y) \sin T y]\left[\cos r_{n} y+2 r_{n} \sin r_{n} y\right] d y \\
\tilde{a}(y)=\frac{1}{2}+\frac{1}{y}+\frac{y}{2 \alpha} .
\end{gathered}
$$


После несложных преобразований, включающих интегрирование по частям, имеем

$$
\begin{aligned}
& S_{1}^{\Gamma}(\alpha, T)=-\frac{1}{\pi} \sum_{r_{n} \geqslant 0}\left[A_{n}^{-}(\alpha, T)-A_{n}^{+}(\alpha, T)\right], \\
& A_{n}^{ \pm}(\alpha, T)=\int_{b_{0}}^{\infty} \frac{e^{-y^{2} /(4 \alpha)}}{y} \sin \left(r_{n} \pm T\right) y d y-\frac{r_{n} \cos \left(r_{n} \pm T\right) b_{0}}{b_{0}\left(r_{n}^{2}+1 / 4\right)}+\frac{1}{2} \frac{\sin \left(r_{n} \pm T\right) b_{0}}{b_{0}\left(r_{n}^{2}+1 / 4\right)} .
\end{aligned}
$$

Формально нужный результат (1.11) получается предельным переходом при $\alpha \rightarrow \infty$ под знаками суммы и интеграла в формуле (5.2). Обоснуем это. Интересующие нас интегралы имеют вид

$$
J(\alpha, \lambda)=\int_{b_{0}}^{\infty} \frac{e^{-y^{2} /(4 \alpha)}}{y} \sin \lambda y d y .
$$

Интегрируя по частям, получаем равенство

$$
\begin{array}{r}
J(\alpha, T)=\frac{\cos \lambda b_{0}}{\lambda b_{0}}+\frac{\sin \lambda b_{0}}{\lambda^{2}}\left(\frac{1}{b_{0}^{2}}+\frac{1}{2 \alpha}\right)-\frac{\sin \lambda b_{0}}{\lambda^{3}}\left(\frac{2}{b_{0}^{3}}+\frac{1}{2 \alpha b_{0}}+\frac{b_{0}}{\alpha^{2}}\right) \\
+\frac{1}{\lambda^{3}} \int_{b_{0}}^{\infty} e^{-y^{2} /(4 \alpha)} \cos \lambda y\left(\frac{6}{y^{4}}+\frac{3}{2} \frac{1}{\alpha y^{2}}\right)+\frac{1}{8 \lambda^{3} \alpha^{3}} J_{2}(\alpha, \lambda),
\end{array}
$$

и в этой формуле

$$
J_{2}(\alpha, \lambda)=\int_{b_{0}}^{\infty} y^{2} e^{-y^{2} /(4 \alpha)} \cos \lambda y d y .
$$

Используя равенство (см. [18])

$$
\int_{0}^{\infty} x^{2} e^{-a x^{2}} \cos b x d x=-\frac{\sqrt{\pi}}{8} \frac{1}{a^{3 / 2}} e^{-b^{2} /(4 a)} H_{2}\left(\frac{b}{2 \sqrt{a}}\right),
$$

где $H_{2}$ - полином Эрмита, имеем оценку

$$
\left|J_{2}(\alpha, \lambda)\right| \leqslant C\left(\lambda^{2} \alpha^{5 / 2} e^{-\lambda^{2} \alpha}+b_{0}^{3}\right) .
$$

Используя эти результаты и разлагая величины $A_{n}^{-}(\alpha, T)-A_{n}^{+}(\alpha, T)$ по обратным степеням $r_{n}$, получим, что

$$
\begin{aligned}
& A_{n}^{-}(\alpha, T)-A_{n}^{+}(\alpha, T) \\
& \quad=2 \frac{\cos r_{n} b_{0}}{r_{n}^{2}}\left[\frac{\cos T b_{0}}{b_{0}} T-\sin T b_{0}\left(\frac{1}{b_{0}^{2}}+\frac{1}{2 \alpha}+\frac{1}{2}\right)\right]+O_{\Gamma}\left(\frac{T^{2}}{r_{n}^{3}}\right)+O_{\Gamma}\left(\frac{1}{r_{n}^{3} \alpha}\right) .
\end{aligned}
$$

Теперь заметим, что при $\beta>2$ (см. [8])

$$
\sum_{r_{n}>1} r_{n}^{-\beta}=C_{\Gamma}<\infty .
$$

Так как в силу формулы Сельберга ряд (5.1) сходится, то из (5.2) и (5.5) следует, что выполняется равенство (1.12). Таким образом, ряд (5.1) сходится равномерно по $\alpha$ при $\alpha>1$ и можно переходить к пределу при $\alpha \rightarrow \infty$ под знаком суммы в (5.2). Возможность такого перехода по знаком $\int_{b_{0}}^{\infty} d y$ следует из формул (5.3), (5.4). 
Теорема и предложение доказаны. При этом функция $F^{\Gamma}(t)$ в равенстве $(1.11)$ имеет вид

$$
F^{\Gamma}(t)=F_{0}^{\Gamma}(t)+F_{1}^{\Gamma}(t),
$$

где функция $F_{0}^{\Gamma}(t)$ определена в $(2.11)$, а функция $F_{1}^{\Gamma}(t)-$ равенствами (4.6)(4.9).

Выше равенство (1.12) и теорема были доказаны одновременно. Покажем, как это равенство выводится из теоремы. Это даст некоторую информацию о характере сходимости ряда в правой части равенства (1.11).

Обозначим этот ряд через $H(t)$ :

$$
H(t)=\frac{1}{\pi} \sum_{r_{n} \geqslant 0}\left[\operatorname{sgn}\left(r_{n}-t\right) \operatorname{si}\left(\left|t-r_{n}\right| b_{0}\right)-\operatorname{si}\left(\left(t+r_{n}\right) b_{0}\right)-\beta_{n}\right] .
$$

В силу теоремы

$$
\mathscr{N}\left(t^{2}+1 / 4\right)=\frac{|F|}{4 \pi}+H(t)+F^{\Gamma}(t) .
$$

Предположим, что $\bar{r}_{p} \leqslant t<\bar{r}_{p+1}$, где $\bar{\lambda}_{p}=\bar{r}_{p}^{2}+1 / 4-$ собственные значения, перенумерованные без учета кратности $\left(\bar{r}_{p}<\bar{r}_{p+1}\right)$, и $p$ достаточно велико. Пусть $\theta>0$, и запишем величину $H(t)$ в виде

$$
H(t)=H_{\theta}(t)+\Delta_{\theta} H(t), \quad \Delta_{\theta} H(t)=\frac{1}{\pi} \sum_{r_{n}>t^{1+\theta}}\left[\operatorname{si}\left(\left(r_{n}-t\right) b_{0}-\operatorname{si}\left(\left(t+r_{n}\right) b_{0}\right)-\beta_{n}\right] .\right.
$$

Используя асимптотическое разложение

$$
-\operatorname{si} x=\frac{\cos x}{x}\left(1-\frac{2 !}{x^{2}}+\frac{4 !}{x^{4}}-\frac{6 !}{x^{6}}+\cdots\right)+\frac{\sin x}{x^{2}}\left(1-\frac{3 !}{x^{2}}+\frac{5 !}{x^{4}}-\frac{7 !}{x^{6}}+\cdots\right),
$$

которое является обертывающим, получим, что

$$
\Delta_{\theta} H(t)=\sum_{r_{n}>t^{1+\theta}} \frac{\cos r_{n} b_{0}}{\pi r_{n}^{2}}\left[-\frac{2 t}{b_{0}} \cos t b_{0}+\frac{2}{b_{0}}\left(\frac{1}{b_{0}}+\frac{1}{2}\right) \sin t b_{0}\right]+O_{\Gamma}\left(t^{1-\theta}\right) .
$$

При выводе этого равенства использовались оценки

$$
t^{2} \sum_{r_{n}>t^{1+\theta}} r_{n}^{-3} \leqslant C_{\Gamma} t^{1-\theta}, \quad t^{3} \sum_{r_{n}>t^{1+\theta}} r_{n}^{-4} \leqslant C_{\Gamma}^{\prime} t^{1-2 \theta} .
$$

Равенство (1.12) теперь следует из (5.7) и сходимости ряда (5.6). Заметим, что (cM. [9])

$$
\sum_{r_{n} \leqslant T} r_{n}^{-2}=O(\ln T)
$$

\section{ЛитературА}

[1] А. Е. Ингам, Распределение простых чисел, Главная редакция общетехнической литературы и номографии, М.-Л., 1936.

[2] H. M. Edwards, Riemann's Zeta-Function, Academic Press, New York, 1974.

[3] А. А. Карацуба, Основъ аналитической теории чисел, Наука, М., 1983.

[4] К. Прахар, Распределение простых чисел, Мир, М., 1967.

[5] Ю. М. Манин, А. А. Панчишкин, Введение в современную теорию чисел, МЦНМО, М., 2009.

[6] С. Ленг, Алгебраические числа, Мир, М., 1966. 
[7] C. J. Moreno, Explicit formulas in the theory of automorphic forms, in: Number Theory Day (Proc. Conf. Rockefeller Univ., New York), Lecture Notes in Math., vol. 626, Springer-Verlag, Berlin, 1977, 73-216.

[8] D. A. Hejhal, The Selberg trace formula and the Riemann zeta-function, Duke Math. J., 43:3 (1976), 441-481.

[9] D. A. Hejhal, The Selberg Trace Formula for $P S L(2, \mathbb{R}), V$. 1, Lecture Notes in Math., vol. 548, Springer-Verlag, Berlin-New York, 1976.

[10] А. Б. Венков, Спектральная теория автоморфных функиий, дзета-функиия Сельберга и некоторые проблемы аналитической теории чисел и математической физики, УМН, 34:3 (1979), 69-135.

[11] B. Randol, A Dirichlet series of eigenvalue type with applications to asymptotic estimates, Bull. London Math. Soc., 13:4 (1981), 309-315.

[12] Д. В. Косыгин, А. А. Минасов, Я. Г. Синай, Статистические свойства спектров операторов Лапласа-Бельтрами на поверхностях Лиувилля, УМН, $48: 4$ (1993), 3-130.

[13] W. Luo, P. Sarnak, Number variance for arithmetic hyperbolic surface, Comm. Math. Phys, 161:2 (1994), 419-432.

[14] S. Geninska, E. Leuzinger, A geometric characterization of arithmetic Fuchsian groups, Duke Math. J., 142:1 (2008), 112-125.

[15] Справочник по специальным бункииям с формулами, графиками и математическими таблицами (ред. М. Абрамовиц, И. Стиган), Наука, М., 1979.

[16] Г. Бейтмен, А. Эрдейи, Высшие трансцендентные функиии. Функции Бесселя. Функиии параболического иилиндра. Ортогональные многочлены, Наука, М., 1966.

[17] В. В. Головчанский, Асимптотика спектралъной функции оператора ЛапласаБельтрами для кокомпактных дискретных подгрупп $S L_{2}(\mathbb{R})$, Матем. сб., 189:7 (1998), 23-36.

[18] А. П. Прудников, Ю. А. Брычков, О. И. Маричев, Интеграль и ряды. Элементарные функции, Наука, М., 1981.

Научно-исследовательский институт

физико-химической биологии им. А. Н. Белозерского,

МГУ им. М. В. Ломоносова
Поступило в редакцию 24 февраля 2011 г. 